\title{
Long term effects of refractory temporal lobe epilepsy on cognitive abilities: a cross sectional study
}

Hennric Jokeit, Alois Ebner

\begin{abstract}
Objective-Intractable epilepsy is related to various transient and chronic brain electric and neurochemical disturbances. There is increasing evidence that chronic epilepsy induces secondary neuronal metabolic and structural decline. However, there is no convincing evidence that the cognitive abilities of patients deteriorate with increasing duration of intractable epilepsy.
\end{abstract}

Methods-To examine whether duration of refractory temporal lobe epilepsy (TLE) is related to generalised cognitive impairment, psychometric intelligence based on the full scale intelligence quotient (FSIQ, WAIS-R) was determined in 209 patients with unilateral TLE. For analyses of variance (ANOVA) patients were grouped into three categories: $<15$, $15-30$, and $>30$ years of refractory TLE.

Results-An ANOVA and a multiple regression analysis showed that duration of TLE affects FSIQ. Patients with $>\mathbf{3 0}$ years of TLE performed worse than patients with 15 or 30 years of TLE. The factors side of seizure origin and type of lesion on MRI did not reach significance. A second ANOVA including education as factor showed that in patients with higher educational attainment, the mean FSIQ was stable for a longer duration of TLE than in less educated patients. Retesting 6 months after anterior temporal lobectomy seizure free patients $(n=85$ of 127) had an higher FSIQ but showed a similar duration effect before and after anterior temporal lobectomy. The variables age at epilepsy onset, education, frequency of interictal epileptiform discharges, frequency of habitual and generalised seizures, serum concentration of antiepileptic drugs, and polypharmacy were statistically controlled.

Epilepsy Centre Epilepsy Surgery Program, Bielefeld, Germany

H Jokeit

A Ebner

Correspondence to: Dr Hennric Jokeit, Klinik Mara 1, Maraweg 21, D-33617 Bielefeld, Germany. Telephone 0049521144 3831 ; fax 0049521144 3553; email jkt@mara.de

Received 10 July 1998 and in final form

8 February 1999

Accepted 16 February 1999
Conclusions-Psychometric intelligence of patients with a longer duration of refractory TLE were most severely impaired. Consequently, refractory TLE seems to be associated with slow but ongoing cognitive deterioration. It is assumed that epilepsy related noxious events and agents exhaust the compensatory capacity of brain functions. However, as in dementia and Alzheimer's disease, higher educational attainment as an indicator of higher brain reserve might delay the cognitive decline. (F Neurol Neurosurg Psychiatry 1999;67:44-50)

Keywords: epilepsy; cognition; dementia
A long duration of intractable epilepsy is related to a considerable number of focal or generalised seizures, pathological interictal electric brain activity, chronic and transient metabolic disturbances, and chronic antiepileptic medication with usually high serum concentrations. ${ }^{1-4}$ It is suggested that these noxious factors may induce secondary neurophysiological and structural long term changes. ${ }^{5-10}$ Still an open question is, however, whether the cognitive abilities of patients deteriorate with increasing duration of intractable epilepsy. A few studies have been aimed at elucidating this question. But neither short term longitudinal nor cross sectional studies showed a convincing relation between psychometric intelligence and the duration of epilepsy in samples of adult patients. ${ }^{11-19}$ On the one hand the absence of an evident duration effect suggests that probably no dramatic cognitive changes occur within periods of some years in adult epileptic patients. On the other hand methodological restrictions-for example, a limited time range of longitudinal studies which rarely exceeds a decade, an undetected cohort bias in cross sectional studies, or confounded variables - might cover possible duration effects. Additionally, in longitudinal studies with small sample sizes the possibility of a type two error is often neglected. Especially, the results of densitometric and volumetric cross sectional studies in patients with temporal lobe epilepsy (TLE) show that differences in these measures became significant only if patients differed in decades of the duration of epilepsy. ${ }^{60-24}$ Moreover, these studies indicate that neuronal injury within and beyond the temporal lobes continues to occur with ongoing seizure activity in patients with TLE. However, it is well known that the brain possesses a large degree of redundancy, plasticity, and compensatory mechanisms that may prevent or postpone a cognitive decline due to small but ongoing brain damage. ${ }^{25} 26$ Epidemiological studies identified that education as an indicator of brain reserve modifies the clinical expression of dementia and Alzheimer's disease. ${ }^{27-31}$ However, the concept of brain reserve or spare capacity has not been considered in studies on cognitive functions of patients with refractory epilepsy.

In the present cross sectional study, we examined the effects of duration of temporal lobe epilepsy on psychometric intelligence as an indicator of global cognitive abilities and integrity of higher brain functions. Furthermore the influence of the variable education on psychometric intelligence and its interaction with the duration 
Clinical and demographical variables with factor duration of epilepsy

\begin{tabular}{|c|c|c|c|}
\hline \multirow[b]{2}{*}{ Variables } & \multicolumn{3}{|l|}{ Duration } \\
\hline & $<15 y$ & $15-30 y$ & $>30 y$ \\
\hline & $n$ & $n$ & $n$ \\
\hline No of subjects & $78(31 \mathrm{~F} / 47 \mathrm{M})$ & $94(45 \mathrm{~F} / 49 \mathrm{M})$ & $37(19 \mathrm{~F} / 18 \mathrm{M})$ \\
\hline TLE (left/right)* & $39 \mathrm{~L}, 39 \mathrm{R}$ & $50 \mathrm{~L}, 44 \mathrm{R}$ & $17 \mathrm{~L}, 20 \mathrm{R}$ \\
\hline Education group $(1 . .5)(\text { low } \leqslant 2 \text {, high } \geqslant 3)^{\star}$ & 38 low, 40 high & 48 low, 46 high & 23 low, 14 high \\
\hline Subjects with only mesiotemporal lesions* & $34(43.6 \%)$ & $47(50 \%)$ & $15(40.5 \%)$ \\
\hline Subjects with generalised seizures in history ${ }^{\star}$ & $54(69.2 \%)$ & $73(77.7 \%)$ & $30(81.1 \%)$ \\
\hline \multicolumn{4}{|l|}{ AED: Subjects with^ } \\
\hline (1) $\mathrm{CBZ}$ & 43 & 39 & 15 \\
\hline (2) $\mathrm{DPH}$ & 14 & 24 & 7 \\
\hline (3) $\mathrm{PB}$ & 18 & 27 & 14 \\
\hline (4) others & 3 & 4 & 1 \\
\hline Mono/polytherapy* & $51 / 27$ & $56 / 38$ & $24 / 13$ \\
\hline \multirow[t]{2}{*}{ Subjects seizure free after ATL ${ }^{\star}$} & $34(70.8 \%)$ & $40(66.7 \%)$ & $11(57.9 \%)$ \\
\hline & Mean (SD) & Mean (SD) & Mean (SD) \\
\hline Age at testing $\dagger$ & $25.4(8.5)$ & $33.3(7.3)$ & $43.8(6.8)$ \\
\hline Age at 1 st seizure $\dagger$ & $13.2(9.9)$ & $9.4(7.4)$ & $5.5(15.8)$ \\
\hline Age at epilepsy onset $†$ & $15.9(9.2)$ & $11.3(7.5)$ & $6.1(5.6)$ \\
\hline Duration of epilepsyt & $9.5(3.7)$ & $22.0(4.1)$ & $37.7(5.9)$ \\
\hline \multirow{2}{*}{ Education $(1-5)^{\star}$} & $2.69(0.88)$ & $2.61(1.0)$ & $2.46(0.9)$ \\
\hline & $\begin{array}{l}\text { Median (25-75 } \\
\text { percentile) }\end{array}$ & $\begin{array}{l}\text { Median (25-75 } \\
\text { percentile) }\end{array}$ & $\begin{array}{l}\text { Median (25-75 } \\
\text { percentile) }\end{array}$ \\
\hline Seizure frequency/month $\ddagger$ & $5(3-11)$ & $7(4-13)$ & $6.5(4-10.6)$ \\
\hline Frequency of secondarily generalised seizures/y & $1(0-1)$ & $1(1-2)$ & $1(1-2.4)$ \\
\hline Frequency of interictal epileptiform discharges $/ 20 \mathrm{~s} \rrbracket$ & $0.167(0.06-0.5)$ & $0.444(0.086-1.66)$ & $0.322(0.068-0.662)$ \\
\hline
\end{tabular}

of epilepsy was investigated. To reduce the number of confounding variables we only considered patients with TLE. Moreover, we examined whether a duration effect persists in patients who became seizure free after anterior temporal lobectomy and whether those patients show improved cognitive abilities.

\section{Patients and methods} PATIENTS

All patients underwent a comprehensive presurgical evaluation. We studied 209 consecutive patients with temporal lobe epilepsy who fulfilled the following criteria: seizures of unilateral temporal origin as shown by continuous interictal and ictal Video/EEG monitoring with scalp and sphenoidal electrodes, unilateral lesions within the temporal lobes as shown by axial and coronal T1 and T2 weighted MRI, and full scale intelligence quotient (FSIQ) greater than 55 to exclude severely mentally impaired patients. Patients had given informed consent that data could be used for research purposes.

CLINICL AND DEMOGRAPHICAL VARIABLES

All clinical and demographical variables are represented in the table. The educational status of the patients was ranked into five categories ranging from leaving school without final qualification (1) to university degree (5). The age at first seizure was determined from the patient's history when the first epileptic seizure or febrile convulsions occurred. The variable age at onset of epilepsy is the age when the epilepsy was manifest. The variable duration of epilepsy reflects the age span from the beginning of habitual seizures to the age at neuropsychological testing. Seizure frequency is the estimated mean frequency of habitual seizures during the year before EEG/video monitoring was performed. The presence of generalised seizures was registered whenever at least one secondarily generalised seizure was reported in a patient's history. Additionally, the absolute frequency within the past year was registered. The frequency of interictal epileptiform discharges was determined during the EEG/video monitoring which preceded the neuropsychological investigation usually by 1 to 6 months. In 96 patients, MRI only showed lesions of the mesiotemporal structures. In 113 patients, temporolateral or basal lesions alone or together with mesial lesions were present. At the time of neuropsychological investigation, the serum concentration of antiepileptic drugs was determined. Because of the variety in antiepileptic medication patients were grouped into four categories; (1) 69 patients had been given anticonvulsive medication with carbamazepine in monotherapy and 28 had carbamazepine in combination with second line drugs, (2) 29 patients had phenytoin monotherapy and 16 had phenytoin in combination with other antiepileptic drugs except phenobarbital, (3) 27 patients had phenobarbital in monotherapy and 32 had phenobarbital in combination with other antiepileptic drugs, (4) eight patients had monotherapy or combinations of second line antiepileptic drugs. Seventy eight patients had antiepileptic drugs in polytherapy and 131 patients had monotherapy. Pharmacoresistance of epilepsy was confirmed in all patients. ${ }^{4}$ Of the 209 patients altogether employed, 127 have undergone anterior temporal lobectomy at present and were neuropsychologically assessed 6 months postoperatively. All patients were on antiepileptic drug treatment 6 months after anterior temporal lobectomy. Eighty five patients who had no seizures except auras within 6 months after anterior temporal lobectomy were considered to be seizure free.

PSYCHOMETRIC MEASURE OF COGNITIVE ABILITIES Patients underwent a neuropsychological evaluation designed for patients with TLE. To 
have a comprehensive psychometric measure of global cognitive abilities which is well matched to age matched healthy controls we used the full scale intelligence quotient (FSIQ) from the German version of the Wechsler adult intelligence scale-revised (WAIS-R). ${ }^{32}{ }^{33}$ The FSIQ was estimated from the subtests information, comprehension, similarities, digit symbol, picture completion, and block design.

STATISTICAL ANALYSES

A data set of 16 independent variables for each patient was evaluated by linear multiple regression analysis. The set included the variables sex, side of seizure origin, age at testing, age at first seizure, age at epilepsy onset, duration of epilepsy, education, ranked seizure frequency, ranked frequency of generalised seizures, presence or absence of generalised seizures in patient's history, ranked frequency of interictal epileptiform discharges, presence or absence of temporal lesions beyond the mesiotemporal structures in MRI, serum concentration of carbamazepine, phenytoin, and phenobarbital, and antiepileptic drug monotherapy or polytherapy. The FSIQ served as the dependent variable. To isolate the variables which significantly contribute to the explanation of variance of the FSIQ a stepwise selection was chosen $\left(\mathrm{p}_{\text {in }}<0.05, \mathrm{p}_{\text {out }}<0.10\right){ }^{34}$

To disclose possible factor interactions and to exclude linear effects of covariates in a one way analysis of variance (ANOVA) model, the continuous variable duration of epilepsy was recoded into three values: $<15$ years $=0,15-30$ years $=1$, and $>30$ years of refractory epilepsy $=2$. The side of seizure origin was submitted as the second factor (left, right). The presence or absence of lesions beyond mesiotemporal structures in MRI was submitted as the third factor $(0,1)$. Education, age at epilepsy onset, ranked frequency of interictal epileptiform discharges, ranked frequency of habitual seizures, presence of secondarily generalised seizures in a patient's history, ranked frequency of secondarily generalised seizures within the past year, serum concentration for carbamazepine, phenytoin, and phenobarbital, and antiepileptic drug monotherapy or polytherapy were controlled as covariates.

As it is known that the variable education explains a considerable amount of variance of FSIQ values, we computed an ANOVA with factors duration of epilepsy $(0,1,2)$ and education (low, high) to disclose possible interactions and to specify the effect of the variable education. The educational level was dichotomised into low (patients who did not attend or finished a secondary school, Realschule in Germany, $n=109$ ) and high (patients who finished at least a secondary school, $n=100$ ). The side of seizure origin, presence or absence of lesions beyond mesiotemporal structures, age at onset of epilepsy, ranked frequency of interictal epileptiform discharges, ranked frequency of habitual seizures, presence of generalised seizures in a patient's history, ranked frequency of secondarily generalised seizures within the past year, serum concentration for carbamazepine, phenytoin, and phenobarbital, and antiepileptic drug monotherapy or polytherapy were controlled as covariates.

A repeated measure ANOVA was computed to test changes in FSIQ after anterior temporal lobectomy as a function of seizure outcome. Then a second repeated measure ANOVA was computed to examine whether a duration effect persisted in patients who became seizure free after anterior temporal lobectomy.

An a priori $\alpha$ level of $p=0.05$ was set for all statistical inference tests and a level of $p=0.15$ for tests of variance homogeneity.

\section{Results}

CLINICAL AND DEMOGRAPHICAL VARIABLES

Clinical and demographical variables are presented in the table. The variables age at testing, age at first seizure, age at epilepsy onset, duration of epilepsy, and ranked frequency of interictal epileptiform discharges differed significantly in groups with $<15$ years, 15 to 30 years, and $>30$ years duration of epilepsy. Patients with longer duration of epilepsy were on average older and had an earlier mean age at onset of epilepsy. Patients with a duration of less than 15 years had fewer interictal epileptiform discharges. No further variable reached significance.

PSYCHOMETRIC INTELLIGENCE MEASURE

Multiple regression analyses on FSIQ disclosed significant contributions by the variables education $(p<0.01 ; \beta=0.543)$ and duration of epilepsy $(p<0.01 ; \beta=-0.195)$ after stepwise selection. The equation explained $34.61 \%$ of total variance (adjusted, $\mathrm{p}<0.01$ ). No further variable contributed significantly. Figure 1 shows a scatter plot of individual FSIQ values against duration of epilepsy. The locally weighted regression curve suggests a negative relation between FSIQ and duration of epilepsy.

Because of the linear dependence of the duration of epilepsy on age at testing and age at onset of seizures, the effect of age at testing on FSIQ cannot be simultaneously controlled with the age at onset of seizure in the ANOVA model. Therefore, the partial correlation between FSIQ and age at testing controlled by duration of epilepsy was determined. Age at testing and FSIQ were not significantly related $(r=0.092, \mathrm{p}=0.184)$.

The ANOVA on FSIQ included three factors; duration of epilepsy $(<15,15-30,>30$ years), side of seizure origin (left, right), and presence or absence of temporal lesions beyond the mesiotemporal structures $(0,1)$. Only the covariate education was significantly related to FSIQ $(p<0.01)$. After removing linear effects of covariates the factor duration of epilepsy was significant $(p<0.01)$. The factors side of seizure onset and presence or absence of temporal lesions beyond mesiotemporal structures did not reach significance. No interactions reached significance.

Post hoc contrasts adjusted by covariates showed that patients with a duration of epilepsy for more than 30 years performed worse than patients with less than 15 years of epilepsy $(p<0.01)$ and patients with 15 to 30 years of 


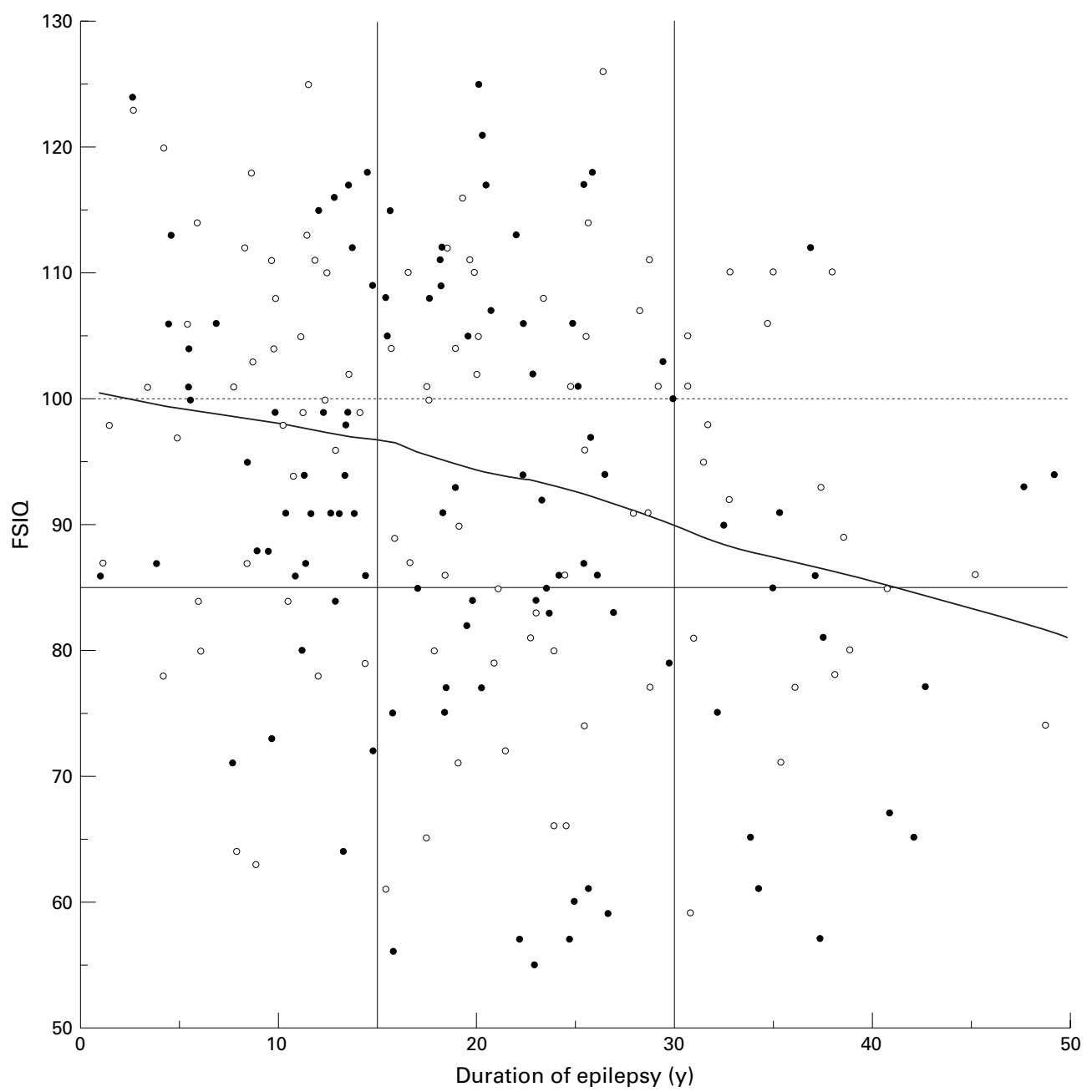

Figure 1 Scatter plot of FSIQ values ( $y$ axis) as a function of duration of epilepsy in years ( $x$ axis). Data from patients with left sided TLE are indicated by filled points, open points represent patients with right sided TLE. To assess the effect of the duration of TLE, data were fitted by a locally weighted regression smoothing, using an iterative weighted least squares method.

epilepsy $(\mathrm{p}<0.05)$. Patients with less than 15 years of epilepsy did not differ from patients with 15 to 30 years in FSIQ.

An ANOVA with factors duration of epilepsy $(0,1,2)$ and education (low, high) was computed to show possible factor interactions. Because of statistical reasons the educational level was dichotomised into low and high educated patients and factors from the former ANOVA side of seizure origin and presence or absence of lesions beyond mesiotemporal structures together with the variables age at epilepsy onset, ranked frequency of interictal epileptiform discharges, ranked frequency of habitual seizures, presence of generalised seizures in a patient's history, ranked frequency of secondarily generalised seizures within the past year, serum concentration for carbamazepine, phenytoin, and phenobarbital, and antiepileptic drug monotherapy or polytherapy served as covariates. The factors education and duration of epilepsy were significant $(p<0.01)$. The interaction between both factors did not reach signifcance $(p=0.14)$. No covariate was significantly related to FSIQ. Figure 2 shows mean FSIQ values for both educational groups as a function of duration of TLE. Contrasts adjusted for covariates showed that the mean FSIQ values of groups with less than 15 years and 15 to 30 years of TLE did not differ in patients with high educational attainment. However, patients with more than 30 years of TLE performed worse than patients with less than 15 years $(p<0.01$, one tailed) or 15 to 30 years of epilepsy $(p<0.01$, one tailed). Patients with low educational attainment and less than 15 years of TLE performed better than patients with 15 to 30 years of TLE ( $p<0.05$, one tailed) and patients with more than 30 years of TLE $(p<0.01$, one tailed). But there was no significant difference between patients with 15 to 30 years and more than 30 years of TLE in the low education group. That comparison might be biased by a floor effect because patients with an FSIQ $<55$ were excluded. Although not confirmed by an ANOVA interaction these contrasts suggest a duration dependent difference in mean FSIQ values of patients with low and high educational attainment. The absence of a significant ANOVA interaction probably results from a similar decremental trend of the duration effect in low and high educated patients. 


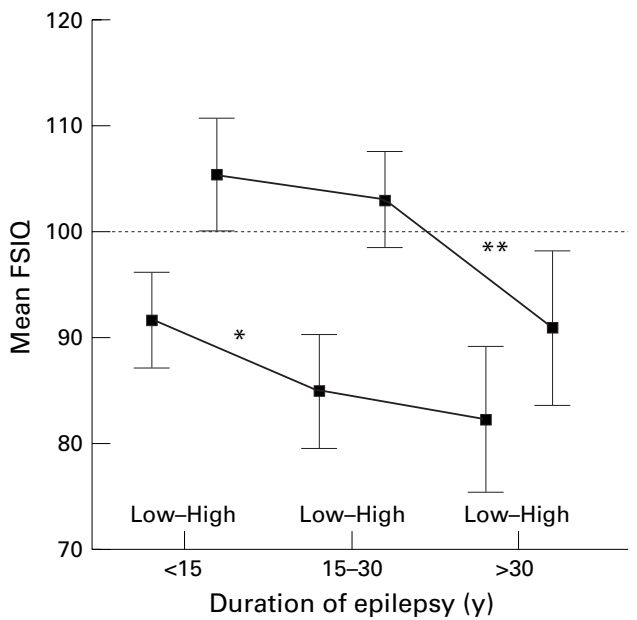

Figure 2 Mean FSIQ values with $95 \%$ confidence intervals ( $95 \% \mathrm{CIs}$ ) of patients with low and high educational attainment for groups with a duration of epilepsy $<15$ years, $15-30$ years, and $>30$ years. Factors education and duration of epilepsy were signifcant $(p<0.01)$. Asterisks $\left({ }^{\star} p<0.05 ;{ }^{\star \star} p<0.01\right.$; one tailed $)$ indicate significant contrasts between adjacent duration groups adjusted for covariates.

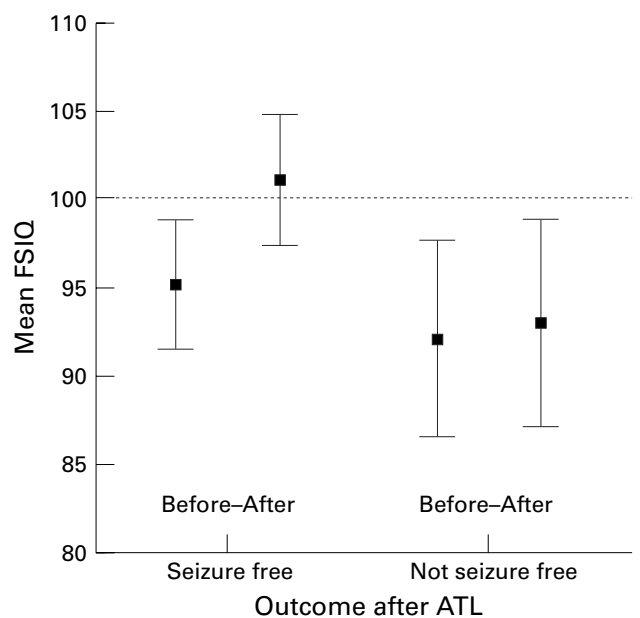

Figure 3 Preoperative (left) and postoperative (right) mean FSIQ values with $95 \%$ CIs of patients who became seizure free or who continued to have seizures. A repeated measures ANOVA showed significantly higher retest mean FSIQ values and an interaction between test-retest change and seizure outcome. Seizure free patients had postoperatively the highest FSIQ.

To show whether the seizure outcome 6 months after ATL influences FSIQ, a repeated measures ANOVA was performed. Education and age at onset of epilepsy served as covariates. Only the factor outcome (seizure free, not seizure free) was considered because the submission of the factor duration of epilepsy $(0,1,2)$ led to inhomogeneity of variance $(\mathrm{p}<0.15)$. Within subjects there was a significant change between test and retest of FSIQ $(p<0.01)$. Mean retest FSIQ values were higher. The factor seizure outcome did not reach significance. However, a significant interaction between test-retest changes and seizure outcome occurred $(p<0.01)$. The covariate education was related to FSIQ $(p<0.01)$. As illustrated in fig 3 seizure free patients improved significantly more than patients with seizures after ATL.

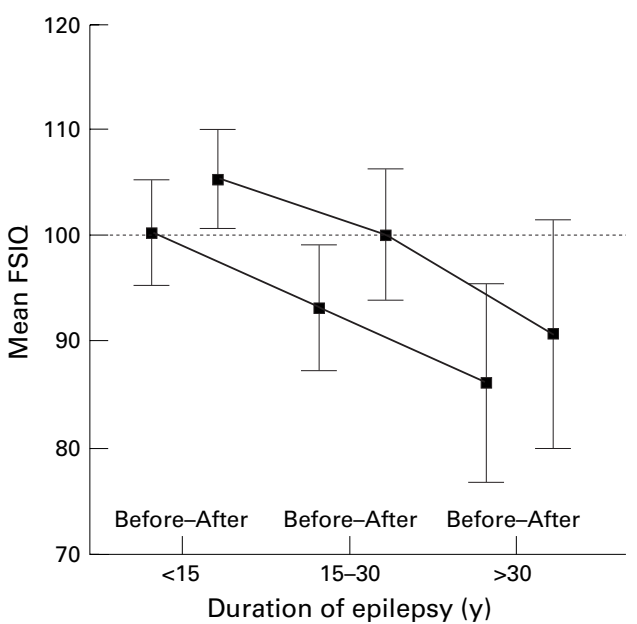

Figure 4 Preoperative (left) and postoperative (right) mean FSIQ values with $95 \%$ CIs of patients who became seizure free for groups with a duration of epilepsy of 15 years, 15-30 years, or $>30$ years. Repeated measures ANOVA showed duration effects $(p<0.01)$ and test-retest changes $(p<0.01)$ but no significant interaction. The duration effect was also present after ATL.

A second repeated measure ANOVA was performed to show whether the duration effect persisted in patients who became seizure free after ATL ( $\mathrm{n}=85)$. The variables education and age at onset of epilepsy served as covariates. Because of the reduced sample size only the factor duration of epilepsy was considered. There was a significant change between test and retest $(p<0.01)$. The factor duration of epilepsy was significant $(p<0.01)$, but we failed to find a significant interaction between test-retest changes in FSIQ and the factor duration of epilepsy $(p=0.62)$. Figure 4 shows the mean preoperative and postoperative FSIQ values with corresponding $95 \%$ CIs as a function of the factor duration of epilepsy. Although postoperative mean FSIQs were consistently higher, the duration effect persisted after ATL.

\section{Discussion}

Our study shows that patients with a long history of intractable TLE were at higher risk of generalised cognitive impairment than patients with a shorter duration of TLE. Comparing patients with higher educational attainment the mean FSIQ was stable for a longer duration of TLE than in groups of less educated patients. In accordance with others we showed that successful surgical treatment of the TLE led to small cognitive improvement compared with patients who continued to have seizures. ${ }^{35} 36$ The duration effect, however, persisted in patients who became seizure free after ATL.

In a first step we showed that the educational level and the duration of epilepsy were the best predictors for psychometric intelligence in our sample. Then we provided evidence that only a long duration of TLE ( $>30$ years) was related to impaired psychometric intelligence in the total sample. The linear influence of the variables age at epilepsy onset, the educational level of patients, the patient's serum concentration of first line antiepileptic drugs, polypharmacy, the frequency of habitual and secondarily generalised seizures, and the frequency of interictal epi- 
leptiform discharges on psychometric intelligence were statistically controlled. Age at testing controlled for duration of TLE was not significantly related to FSIQ. The factors side of TLE and presence or absence of lesions beyond the mesiotemporal structures did not show main effects or interactions. Therefore, the effect of duration of epilepsy cannot be attributed to those covariates and factors. The patient's educational level captured the most amount of FSIQ variance. This could explain why, by contrast with other studies, the variable age at onset of epilepsy did not reach significance. ${ }^{18}$ Age at onset of epilepsy is related to duration of epilepsy and academic achievement of adult patients. An early onset of refractory epilepsy considerably affects school success. On the other hand academic achievement is related to psychometric intelligence in normal subjects and epileptic patients. Therefore, the variable age at onset of epilepsy shares variance from different sources. If these sources of variance are statistically controlled, the remaining variance might be too small to result in significant effects of the variable age at onset of epilepsy.

It is reasonable to assume that human brains develop a functional reserve or have a spare capacity to cope with a stepwise neuronal loss by efficiency, redundancy, plasticity, and reorganisation. ${ }^{25} 3738$ Studies on different degenerative brain disorders (for example, Parkinson disease, vascular, and Alzheimer's dementia) suggest that a functional decline becomes apparent only if a certain amount of brain parenchyma is insulted. ${ }^{39-43} \mathrm{~A}$ long duration of intractable TLE is related to a considerable number of focal or secondarily generalised seizures, pathological interictal electric brain activity, chronic and transient metabolic disturbances due to morphological lesions, seizures, and antiepileptic medication, and chronic antiepileptic medication with usually high serum concentrations. ${ }^{1-4}$ It is suggested that each of these factors may separately cause adverse cognitive functioning. ${ }^{45}$ The presence of reactive microglia, ${ }^{7}$ reduced dendritic spine density, dendritic swellings, ${ }^{6}$ and senile plaques ${ }^{46}$ in anterior temporal lobectomy specimens suggests that neuronal injury continues to occur with ongoing seizure activity in patients with TLE. Multani et al first showed a correlation between decreased dendritic spine density remote from the epileptogenic zone and duration of seizure history. ${ }^{6}$ In patients with mesial TLE densitometric techniques showed secondary declines in hippocampal neuron densities with long histories of habitual seizures. ${ }^{204748}$ Recent studies suggested a secondary decline of hippocampal volume and temporal lobe metabolism in patients with refractory TLE. ${ }^{21-24}$ Hermann et al reported that patients with hippocampal sclerosis had more generalised cognitive impairment, a significant longer history of intractable TLE, and a lower educational level than patients with TLE without significant hippocampal sclerosis. ${ }^{49}$ We assume that a cumulation of small neurodegenerative effects of noxious neurochemical agents, abnormal brain electric events, and metabolic disturbances over decades of epi- lepsy accompanied by aging may increase the probability that the functional brain reserve or spare capacity is exhausted at a surprisingly young age (mean age of patients with TLE duration $>30$ years was 44 years) and deterioration of cognitive functions may begin. ${ }^{37} 3850$ The extent of functional reserve and therefore the vulnerability of brain functions may vary considerably between persons. It was suggested that higher educational attainment is related to a higher reserve against cognitive impairment due to stepwise ongoing brain injury. ${ }^{29}{ }^{51}$ Our results of analyses in patients with lower and higher educational attainment are in accordance with findings of epidemiological studies on dementia and Alzheimer's disease. ${ }^{27} 283031$ In patients with higher educational attainment the mean FSIQ was stable for a longer duration of TLE than in less educated patients. Higher educational attainment as an indicator of higher cognitive reserve might delay the onset of cognitive decline in patients with intractable TLE.

Finally, we showed that patients who underwent anterior temporal lobectomy and became seizure free after surgery had a small improvement in psychometric intelligence. However, the effect of duration of epilepsy persisted in patients who became seizure free after anterior temporal lobectomy. This finding suggests that successful treatment of TLE does not compensate the difference in FSIQ between patients with longer and shorter duration of epilepsy. Thus the observed duration in differences in FSIQ may represent a permanent impairment of cognitive abilities. None the less, independent of the duration of epilepsy, patients who became seizure free improved more than patients who continued to have seizures.

Although we found duration dependent differences in the frequency of interictal epileptiform discharges, neither the frequency of these discharges nor the frequency of seizures were significantly related to FSIQ values. The recent discovery by Savic et al that brain metabolic disturbances are correlated with seizure semiology suggests that the spread pattern of seizure activity might affect brain functioning. ${ }^{3} 10$ Additionally, the duration of seizure activity might be of relevance..$^{52}$ Thus, frequency, spread pattern, and duration of seizures together rather than frequency alone may be associated with the extent of functional deficits. $^{53}$

The fact that we found significant effects in the whole sample analysis only in patients with a history of intractable epilepsy lasting longer than three decades may question conclusions drawn from negative findings of studies on adverse effects of duration of refractory TLE. ${ }^{17}{ }^{54}$ Based on our results only prospective long term studies which exceed three decades might disclose the causes of a presumable decline in cognitive functioning of patients with intractable TLE. Such studies may solve the important question whether certain epileptic syndromes are progressive disorders. ${ }^{455-59}$ However, today well controlled cross sectional studies comparing different well defined epileptic syndromes and including retest measures to 
compare different treatment strategies may help to isolate adverse factors.

Although we cannot definitely exclude the possibility that our results were biased by undetected cohort effects the present data might suggest that an early and radical treatment of TLE could preserve normal cognitive functioning in patients with refractory TLE.

We thank the many members of the Bethel Epilepsy Surgery Program, who have consistently collected and maintained the patient data files, and Professor Markowitsch, Dr May, Professor Seitz, Professor Wallesch, and Professor Wolf for helpful suggestions and comments on the manuscript.

1 Theodore WH, Bromfield E, Onorati L. The effect of carbamazepine on cerebral glucose metabolism. Ann Neurol 1989;25:516-20

2 Arnold S, Schlaug G, Niemann H, et al. Topography of interictal glucose hypometabolism in unilateral mesiotemporal epilepsy. Neurology 1996;46:1422-30.

3 Savic I, Altshuler L, Baxter L, et al. Pattern of interictal hypometabolism in PET scans with fludeoxyglucose F 18 reflects prior seizure types in patients with mesial temporal lobe seizures. Arch Neurol 1997;54:129-36.

4 Hermanns G, Noachtar S, Tuxhorn I, et al. Systematic testing of medical intractability for carbamazepine, phenytoin, and phenobarbital or primidone in monotherapy for patients considered for epilepsy surgery. Epilepsia 1996;37: patients

5 Ben-Ari Y, Represa A. Brief seizure episodes induce long-term potentiation and mossy fibre sprouting in the long-term potentiation and mossy fibre sp
hippocamus. Trends Neurosci 1990;13:312-8.

6 Multani P, Myers RH, Blume HW, et al. Neocortical dendritic pathology in human partial epilepsy: a quantitative Golgi study. Epilepsia 1994;35:728-36.

7 Beach TG, Woodhurst WB, MacDonald DB, et al. Reactive microglia in hippocampal sclerosis associated with human temporal lobe epilepsy. Neurosci Lett 1995;191:27-30.

8 Marsh L, Morrell MJ, Shear PK, et al. Cortical and hippocampal volume deficits in temporal lobe epilepsy. Epilepsia 1997;38:576-87.

9 Bengzon J, Kokaia Z, Elmer E, et al. Apoptosis and proliferation of dentate gyrus neurons after single and intermittent limbic seizures. Proc Natl Acad Sci U S A 1997;94:10432-7.

10 Jokeit H, Seitz RJ, Markowitsch HJ, et al. Prefrontal asymmetric interictal glucose hypometabolism and cognitive impairment in patients with temporal lobe epilepsy. Brain 1997;120:2283-94.

11 Seidenberg M, O'Leary DS, Berent S, et al. Changes in seizure frequency and test-retest scores on the Wechsler adult zure frequency and test-retest scores on the
intelligence scale. Epilepsia 1981;22:75-83.

12 Bourgeois BFD, Prensky AL, Palkes HS, et al. Intelligence in epilepsy: a prospective study in children. Ann Neurol 1983; 14:438-44.

13 Rodin EA, Schmaltz S, Twitty G. Intellectual functions of patients with childhood-onset epilepsy. Dev Med Child Neurol 1986;28:25-33.

14 Brown SW, Vaughan M. Dementia in epileptic patients. In Trimble MR, Reynolds EH, eds. Epilepsy, behaviour and cognitive function. Chichester: John Wiley, 1988:177-88.

15 Trimble MR. Cognitive hazards of seizure disorders. Epilepsia 1988;29(suppl 1):S19-24.

16 Dodrill CB, Wilensky AJ. Neuropsychological abilities before and after 5 years of stable antiepileptic drug therapy. Epilepsia 1992;33:327-34

17 Selwa LM, Berent S, Giordani B, et al. Serial cognitive testing in temporal lobe epilepsy: longitudinal changes with medical and surgical therapies. Epilepsia 1994;35:743-9.

18 Strauss E, Loring D, Chelune G, et al. Predicting cognitive impairment in epilepsy: findings from the Bozeman Epilepsy

19 Brown SW. Epilepsy dementia: intellectual deterioration as a consequence of epileptic seizures. Epilepsia 1996; 37(suppl 4):S122-3.

20 Mathern GW, Babb TL, Pretorius JK, et al. The pathophysiologic relationships between lesion pathology, intracranial ictal EEG onsets, and hippocampal neuron losses in temporal lobe epilepsy. Epilepsy Res 1995;21:133-47.

21 Barr WB, Ashtari M, Schaul N. Bilateral reductions in hippocampal volume in adults with epilepsy and a history of febrile seizures. F Neurol Neurosurg Psychiatry 1997;63:4617.

22 Breier JL, Mullani NA, Thomas AB, et al. Effects of duration of epilepsy on the uncoupling of metabolism and blood flow in complex partial seizures. Neurology 1997;48: 1047-53.

23 Salmenperä T, Kälviäinen R, Partanen K, et al. Hippocampal damage caused by seizures in temporal lobe epilepsy. Lancet 1998:351:35.

24 Jokeit H, Ebner A, Arnold S, et al. Bilateral depressions of hippocampal volume, glucose metabolism, and Wada hemispheric memory performance are related to the duration of mesial temporal lobe epilepsy. Epilepsia 1998 39(suppl 2):35
25 Lewin R. Is your brain really necessary? Science 1980;210: $1232-4$

26 Calne DB, Eisen A, McGeer E, et al. Alzheimer's disease, Parkinson's disease, and motoneurone disease: abiotrophic interaction between ageing and environment? Lancet 1986; i(8515): 1067-70

27 Zhang M, Katzman R, Salmon D, et al. The prevalence of dementia and Alzheimer's disease in Shanghai, China: impact of age, gender, and education. Ann Neurol 1990;27: $428-37$

28 Stern Y, Gurland B, Tatemichi TK, et al. Influence of education and occupation on the incidence of Alzheimer's disease. FAMA 1994;271:1004-10.

29 Satz P. Brain reserve capacity on symptom onset after brain njury: a formulation and review of evidence for threshold theory. Neuropsychology 1993; 7:273-95.

30 Evans DA, Hebert LE, Becket LA, et al. Education and other measures of socioeconomic status and risk of incident Alzheimer disease in a defined population of older persons. Arch Neurol 1997;54:1399-405.

31 Schmand B, Smit JH, Geerlings MI, et al. The effects of intelligence and education on the development of demenia. A test of the brain reserve hypothesis. Psychol Med 997;27:1337-44

32 Wechsler D. WAIS-R manual. New York: The Psychological Corporation, 1981

33 Tewes U. Manual des Hamburg-Wechsler Intelligenztest für Erwachsene Revision 1991. Bern: Verlag Hans Huber, 1991.

34 Norusis MJ. SPSS/PC+ Statistics 4.0. Chicago: SPSS, 1990.

35 Novelly RA, Augustine EA, Mattson RH, et al. Selective memory improvement and impairment in temporal lobectomy for epilepsy. Ann Neurol 1984;15:64-7.

36 Ivnik JR, Sharbrough FW, Laws ER. Anterior temporal lobectomy for control of partial complex seizures: information for counseling patients. Mayo Clin Proc 1988;63: 783-91.

37 Meier-Ruge W, Hunziker O, Iwangoff P. Senile dementia: a threshold phenomenon of normal aging? A contribution to the functional reserve hypothesis of the brain. Ann NY Acad Sci 1991;621:104-18.

38 Stern RA, Silva SG, Chaisson N, et al. Influence of cognitive reserve on neuropsychological functioning in asymptomatic human immunodeficiency virus-1 infection. Arch Neurol 1996;53:148-53.

39 Tomlinson BE, Blessed G, Roth M. Observations on the brains of demented old people. F Neurol Sci 1970;11:205-42.

0 Hornykiewicz O. Neurochemical pathology and the etiology of Parkinson disease. Mt Sinai f Medicine 1988;55:11-20.

41 Boone KB, Miller BL, Lesser IM, et al. Neuropsychological correlates of white-matter lesions in healthy elderly subjects. Arch Neurol 1992;49:549-54.

42 Small GW, Mazziota JC, Collins MT, et al. Apolipoprotein E type 4 allele and cerebral glucose metabolism in relatives at risk for familial Alzheimer disease. FAMA 1995;273:942-7.

43 Pasquier F, Leys D. Why are stroke patients prone to develop dementia? $\mathcal{F}$ Neurol 1997;244:135-42.

44 Dreifuss EF. Cognitive function: victim of disease or hostage to treatment? Epilepsia 1992;33 (suppl 1):S7-12.

45 Lesser RP, Lüders H, Wyllie E, et al. Mental deterioration in epilepsy. Epilepsia 1986;27(suppl 2):S105-23.

16 Mackenzie IRA, Miller LA. Senile plaques in temporal lobe epilepsy. Acta Neuropathol 1994;87:504-10.

47 Mathern GW, Babb TL, Vickrey BG, et al. The clinicalpathogenic mechanism of hippocampal neuron loss and surgical outcomes in temporal lobe epilepsy. Brain 1995;118:105-18.

48 Mathern GW, Babb TL, Leite JP, et al. The pathogenic and progressive features of chronic human hippocampal epilepsy. Epilepsy Res 1996;26:151-61.

49 Hermann BP, Seidenberg M, Schoenfeld J, et al. Neuropsychological characteristics of the syndrome of mesial temporal lobe epilepsy. Arch Neurol 1997;54:369-76.

50 Mori E, Hirono N, Yamashita H, et al. Premorbid brain size as a determinant of reserve capacity against intellectual decline in Alzheimer's disease. Am f Psychiatry 1997;154: 18-24.

51 Timiras PS. Education, homeostasis, and longevity. Exp Gerontol 1995;30:189-98.

52 Olney JW, Collins RC, Sloviter RS. Excitotoxic mechanisms of epileptic brain damage. In: Delgado-Escueta AV, Ward AAJ, Woodbury DM, et al, eds. Basic mechanisms of the epilepsies. New York: Raven Press, 1986:857-77.

53 Lüders HO, Awad I. Conceptual considerations. In: Lüders HO, ed. Epilepsy surgery. New York: Raven Press, 1991:5162 .

4 Mackenzie IRA, McLachlan RS, Kubu CS, et al. Prospective neuropsychological assessment of nondemented patients with biopsy proven senile plaques. Neurology 1996;46:425-9.

55 Sutula T, Cascino G, Cavazos J, et al. Mossy fiber synaptic reorganization in the epileptic human temporal lobe. Ann Neurol 1989;26:321-30.

56 Gloor P. Mesial temporal sclerosis: historical background and an overview from a modern perspective. In: Lüders HO, ed. Epilepsy surgery. New York: Raven Press, 1991:689-703.

57 Girvin JP. Is epilepsy a progressive disorder? $\mathcal{f}$ Epilepsy

58 Mathern GW, Pretorius JK, Babb TL. Influence of the type of initial precipitating injury and at what age it occurs on course and outcome in patients with temporal lobe seizures. F Neurosurg 1995;82:220-7.

59 Sadzot B. Epilepsy: a progressive disease? BMF 1997;314: $391-2$. 